\title{
Eficacia del Plan de Cinco Días para Dejar de Fumar en una ciudad de Argentina
}

\author{
Mariana Elizabeth Alfaro ${ }^{1}$ y Marcelo Ricardo Simi ${ }^{1}$
}

Forma de citar Alfaro ME, Simi MR. Eficacia del Plan de Cinco Días para Dejar de Fumar en una ciudad de Argentina. Rev Panam Salud Publica. 2007;21(5):301-6.

RESUMEN Objetivos. Evaluar la eficacia del Plan de Cinco Días para Dejar de Fumar (P5D) al final de la intervención y al año, según las características de los asistentes a los cursos de este plan realizados en la ciudad de Rosario, Santa Fe, Argentina.

Métodos. Estudio cuasiexperimental, longitudinal, prospectivo y comparativo basado en la evaluación de ocho cursos del P5D en la ciudad de Rosario, Santa Fe, Argentina. Participaron 739 personas (50,5\% de mujeres y 49,5\% de hombres). La eficacia al final de los cursos y al año se evaluó en una muestra de 281 participantes. La eficacia se relacionó con la edad de comienzo del tabaquismo, el consumo promedio diario de cigarrillos, los años de consumo, el nivel educacional, el número de intentos previos de abandonar el hábito de fumar y el nivel de dependencia a la nicotina según la escala de Fagerström. La asociación entre las variables cualitativas se determinó mediante el riesgo relativo (RR) y su intervalo de confianza de 95\% (IC95\%). Las diferencias entre las medias de las variables cuantitativas se determinaron mediante la prueba de la t para muestras independientes y se empleó la prueba de la ji al cuadrado para establecer las diferencias entre las variables categóricas estudiadas.

Resultados. De los 281 participantes de la muestra para medir la eficacia, 201 (71,5\%) habian dejado de fumar al finalizar los cursos, de ellos 104 (51,7\%) eran hombres y 97 (48,3\%) eran mujeres. Al cabo del año, $77(27,4 \%$ ) participantes de la muestra se mantenían sin fumar (48 hombres [34,8\% de los hombres de la muestra] y 29 mujeres [20,3\% de las mujeres de la muestra], $\left.\chi^{2}=10,537, P=0,001\right)$. Se encontró una asociación significativa entre la recaída en el tabaquismo por una parte y el sexo femenino $(R R=1,22 ; \mathrm{P}=0,005)$ y un valor en la prueba de dependencia a la nicotina igual o mayor que $7(R R=1,17 ; \mathrm{P}=0,03)$, por el otro. El haber comenzado a fumar antes de los 20 años resultó un factor protector contra la recidiva del tabaquismo en los asistentes a los P5D. Los factores condicionantes más frecuentes de la recidiva fueron la presencia de sintomas del síndrome de abstinencia (40,3\%) y las situaciones estresantes (28,2\%). Conclusiones. El P5D es una herramienta eficaz de prevención secundaria del tabaquismo y en la población estudiada, su eficacia estuvo asociada con el sexo y el nivel de dependencia a la nicotina.

Palabras clave Cese del tabaquismo, Argentina.

1 Cátedra de Medicina Preventiva, Carrera de Medicina, Facultad de Ciencias de la Salud, Universidad Adventista del Plata. La correspondencia se debe dirigir a Marcelo R. Simi, Facultad de Ciencias de la Salud, Universidad Adventista del Plata, Calle 25 de Mayo No. 99 (3103), Villa Libertador General San Martín, Entre Ríos, Argentina. Correo electrónico: marcelosimi@doc.uap.edu.ar
El tabaquismo - un problema que tiene grandes repercusiones sanitarias, ambientales, sociales, políticas y económicas- está relacionado con alrededor de $80 \%$ de los casos de enfermedad pulmonar obstructiva crónica y es una de las principales causas prevenibles de muerte en todo el mundo $(1,2)$. Este nocivo hábito es responsable de aproximadamente $30 \%$ de los fallecimientos por cáncer y de $20 \%$ de los atribuidos a enfermedades cardio- 
vasculares. Según la Organización Mundial de la Salud (OMS), el número de muertes provocadas por el tabaquismo puede aumentar de alrededor de 4 millones de defunciones anuales en la actualidad, a 8,4 millones anuales en el año 2020 (3).

En la Región de las Américas, este hábito también constituye uno de los desafíos de salud pública más importantes, ya que causa al menos 845000 defunciones por año (4). Argentina es el segundo país con mayor proporción de fumadores en América Latina y el Caribe y el mayor consumidor de tabaco. A la población argentina le corresponde $15 \%$ del total del tabaco consumido en esta área, a pesar de que en ese país vive solamente $7 \%$ de la población latinoamericana y caribeña.

Según las estadísticas nacionales argentinas, aproximadamente $40 \%$ de los adultos fuman y unas 40000 personas fallecen anualmente en ese país por causas relacionadas con el tabaquismo (5). Según datos de la Sociedad Argentina de Cardiología, alrededor de nueve millones de adultos son adictos al cigarrillo y la época más frecuente de inicio de ese hábito es la adolescencia (6). La prevalencia de tabaquismo en las mujeres argentinas ha aumentado considerablemente: en 1992 constituían 23\% del total de los fumadores del país (7) y diez años después eran el 66\% (8).

Una de las primeras estrategias empleadas para la prevención secundaria del tabaquismo es el Plan de Cinco Días para Dejar de Fumar (P5D) (9), desarrollado en 1960 por la Iglesia Adventista del Séptimo Día y aplicado ya en numerosos países de todo el mundo $(10,11)$. Según estudios realizados en diversos países, la eficacia del P5D al año varía entre $16 \%$ (12) y 35\% (13). Sin embargo, no se cuenta con una evaluación objetiva sobre la eficacia del P5D en Argentina.

El objetivo del presente estudio fue evaluar la eficacia del P5D al final de la intervención y al año, según las características de los asistentes a los cursos de este plan realizados en la ciudad de Rosario, Santa Fe, Argentina.

\section{MATERIALES Y MÉTODOS}

En este estudio se utilizó un diseño metodológico cuasiexperimental, longitudinal, prospectivo y comparativo basado en la evaluación de ocho cursos del P5D llevados a cabo de forma simultánea en diferentes zonas (norte, centro y sur) de la ciudad de Rosario, provincia de Santa Fe, Argentina. Los cursos se desarrollaron durante los meses de septiembre (en las tres zonas), octubre (en las tres zonas) y noviembre (solamente en las zonas centro y sur) de 2004 y la evaluación se llevó a cabo inmediatamente y al año de terminar cada curso.

En total, 739 personas asistieron a los cursos del P5D, de ellos, 373 (50,5\%) eran mujeres y 366 (49,5\%) eran hombres. Todos se inscribieron en los cursos voluntaria y gratuitamente, incentivados por anuncios publicitarios que aparecieron en varios medios de información masiva.

La eficacia de la intervención se evaluó en una muestra de 281 participantes, seleccionados mediante un método aleatorio simple, estratificado según el sexo y la zona donde se realizaron los cursos. El tamaño de la muestra se calculó a partir de una proporción esperada de $25 \%$ y una precisión de $96 \%$ y su homogeneidad se determinó mediante la prueba de la $t$. No se encontraron diferencias significativas entre la población de estudio y la muestra en cuanto al género $(P=0,460)$, el número de intentos previos de abandonar el hábito de fumar $(P=0,937)$ y el lugar del curso $(P=0,954)$, según la prueba de la ji al cuadrado. Si bien se encontró diferencia significativa en el número de años de consumo, la media de ese valor es más elevada en el grupo de la muestra, por lo que no interfiere en el diseño del estudio. En el cuadro 1 se muestran los resultados del análisis de las variables cuantitativas de la población de estudio y la muestra.

El P5D es un programa estandarizado de cinco sesiones de aproximadamente 90 minutos cada una en cinco noches consecutivas. Cada encuentro consta de: una sección médica (provee información de salud pública, explica los efectos neuroendocrinológicos producidos por la nicotina en el sistema nervioso central y los efectos fisiopatológicos del tabaquismo en los sistemas respiratorio y cardiovascular, con especial énfasis en los beneficios de abandonar el tabaquismo); una sección de psicología, basada en el trabajo cognitivo y conductual de grupo, cuyo propósito es reforzar la motivación y la voluntad; una sección de técnicas antiestrés y de relajación, basadas en el descanso nocturno, el ejercicio físico al aire libre y una dieta bien equilibrada rica en frutas y verduras, con abundante agua y sin bebidas cafeinadas o alcohólicas. No se utilizaron métodos antitabáquicos farmacológicos o de otro tipo.

Los datos de los participantes se obtuvieron mediante un formulario autoaplicado con un diseño estructurado de preguntas. Este cuestionario se entregó al comenzar la primera sesión del P5D y se recogió en la segunda sesión.

Para la construcción de los intervalos de edad se utilizó la regla de Sturges, de manera que cada intervalo quedara representado proporcionalmente (14). Los cursos se agruparon según las tres zonas de la ciudad que se diferencian por su nivel socioeconómico: la zona norte, de mejor nivel socioeconómico; la zona central, de nivel intermedio; y la sur, de nivel socioeconómico más bajo. El nivel educacional se clasificó en tres categorías, de acuerdo con el número de años de educación formal terminados: básico, hasta 7 años; medio, de 8 a 12 años; y alto, más de 12 años. Para el análisis asociativo, estas categorías se reagruparon en dos grupos: bajo nivel de estudios formales (hasta 7 años) y alto nivel de estudios formales (más de 7 años).

Las características de la adicción al tabaquismo se describieron mediante los siguientes indicadores:

- Edad de comienzo del tabaquismo, en años cumplidos.

- Consumo diario de tabaco, en número promedio de cigarrillos por día: hasta 20 cigarrillos por día o más de 20 cigarrillos por día. 
- Años totales de consumo, en años: menos de 20 años y 20 años o más.

- Nivel de dependencia a la nicotina según la prueba de Fagerström (15). Se clasificó en tres niveles: leve (puntaje de 0 a 3), moderada (de 4 a 6) y alta (de 7 a 10) (16). Para realizar el análisis asociativo se reagruparon en dos niveles: bajo (puntaje de 0 a 6) y alto (de 7 a 10).

- Número de intentos previos de abandonar el tabaquismo. Se consideraron dos categorías: ningún intento previo y lo intentó anteriormente.

La eficacia del P5D se definió mediante dos indicadores: el abandono inmediato del consumo de tabaco por parte del participante y la abstinencia al año de terminado el curso. Los datos para determinar la eficacia al año se obtuvieron mediante una entrevista telefónica estructurada en la que si procedía también se preguntaron las causas de la reincidencia en el tabaquismo y si el participante tuvo apoyo social y familiar en su intento.

La asociación entre las variables cualitativas se determinó mediante el riesgo relativo (RR) y su intervalo de confianza de 95\% (IC95\%). Las diferencias entre las medias de las variables cuantitativas se determinaron mediante la prueba de la $t$ para muestras independientes y se empleó la prueba de la ji al cuadrado para establecer las diferencias entre las variables categóricas estudiadas. El nivel de significación fue de $P<0,05$.

\section{RESULTADOS}

\section{Características de los participantes}

La edad promedio de los asistentes al P5D fue de 44,2 años (IC95\%: 44 a $45,8)$ con una distribución de frecuencias cercana a la normal. El grupo de edad más numeroso entre los participantes en el P5D fue el de 36 a 56 años. La zona con mayor participación fue el centro, con $344(46,5 \%)$ participantes, seguida de la zona sur, con 246 (33,3\%) participantes, y la zona norte con 149 $(20,2 \%)$.

CUADRO 1. Descripción de las características personales y de la adicción de los asistentes al Plan de Cinco Días para Dejar de Fumar, según el grupo al que pertenecen, Rosario, Argentina, 2004

\begin{tabular}{|c|c|c|c|c|c|}
\hline \multirow[b]{2}{*}{ Características } & \multicolumn{2}{|c|}{ Población de estudio } & \multicolumn{2}{|c|}{ Muestra } & \multirow[b]{2}{*}{$P^{b}$} \\
\hline & Media & $\mathrm{IC} 95 \%^{\mathrm{a}}$ & Media & IC95\% & \\
\hline Edad & 44,2 & $43,3-45,1$ & 46,0 & $44,6-47,7$ & 0,062 \\
\hline Nivel educacional, años de estudio formal & 12,9 & $12,6-13,3$ & 13,0 & $12,4-13,7$ & 0,982 \\
\hline $\begin{array}{l}\text { Edad de comienzo del tabaquismo, } \\
\text { años cumplidos }\end{array}$ & 17,2 & $16,7-17,4$ & 17,0 & $16,4-17,4$ & 0,638 \\
\hline $\begin{array}{l}\text { Consumo promedio diario de cigarrillos, } \\
\text { unidades }\end{array}$ & 24,6 & $23,4-25$ & 24,0 & $22,7-25,1$ & 0,422 \\
\hline Tiempo de tabaquismo, años & 25,9 & $25,1-26,8$ & 27,8 & $26,4-29,1$ & 0,025 \\
\hline Nivel de dependencia ${ }^{c}$ & 5,1 & $4,9-5,3$ & 5,1 & $4,8-5,3$ & 0,743 \\
\hline
\end{tabular}

a IC95\%: intervalo de confianza de 95\%.

b Para la prueba de la $t$. Nivel de significación: 0,05.

c Puntuación según la prueba de Fagerström (15).

Con relación al nivel educacional de los participantes, el promedio de años de estudios formales fue de 12,9 (C195\%: 12,6 a 13,3), con un predominio de personas con nivel educacional alto $(364 ; 49,3 \%)$ y medio $(269 ; 36,4 \%)$.

\section{Características de la adicción}

La mayoría de los asistentes al P5D comenzaron a consumir tabaco en la adolescencia y en promedio consumían poco más de 20 cigarrillos por día (cuadro 1). En general, 707 (95,7\%) de ellos consumían entre 20 y 40 cigarrillos por día.

La dependencia promedio a la nicotina de los asistentes fue moderada (5 puntos en la escala de 10); $327(44,2 \%)$ del total tenían un nivel moderado de dependencia, $216(29,2 \%)$ un nivel alto y $196(26,5 \%)$ un nivel de dependencia bajo.

En lo que respecta al número de intentos previos, $227(30,7 \%)$ de los participantes intentaron abandonar el tabaco anteriormente al menos tres veces y $154(20,8 \%)$ nunca lo había intentado antes.

\section{Eficacia del Plan de Cinco Días}

Al finalizar los cursos del P5D, de los 281 participantes de la muestra (138 hombres y 143 mujeres), 201 (71,5\%) habían dejado de fumar. De ellos, 104
(51,7\%) eran hombres y $97(48,3 \%)$ eran mujeres (figura 1). Al cabo de un año, $77(27,4 \%)$ participantes de la muestra se mantenían sin fumar (conformados por 48 hombres [34, $8 \%$ de los hombres de la muestra] y 29 mujeres [20,3\% de las mujeres de la muestra], $\chi^{2}=10,537$, $\mathrm{P}=0,001)$. Como se observa en la figura 1, los hombres representaron una proporción de los abstinentes al año significativamente mayor $(62,3 \%)$ que las mujeres $\left(37,7 \% ; \chi^{2}=7,425, \mathrm{P}=0,005\right)$.

Se encontró una asociación significativa entre la recaída en el tabaquismo por una parte y el sexo femenino (RR $1,22 ; P=0,005)$ y un valor en la prueba de dependencia a la nicotina igual o mayor que 7 (RR 1,17; $P=0,03$ ), por la otra. El haber comenzado a fumar antes de los 20 años resultó un factor protector contra la recidiva del tabaquismo en los asistentes a los P5D (cuadro 2). Los factores condicionantes más frecuentes de la recidiva fueron la presencia de síntomas del síndrome de abstinencia $(40,7 \%)$ y las situaciones estresantes (28,5\%) (cuadro 3).

No se observaron diferencias significativas entre los resultados obtenidos según la zona de asistencia al P5D $(P=0,398)$.

\section{DISCUSIÓN}

El grupo de participantes que asistió a los cursos del P5D estuvo conformado por personas de edad media, 
FIGURA 1. Abstinencia inmediata y al año en una muestra de los participantes en el Plan de Cinco Días para Dejar de Fumar, Rosario, Argentina, septiembre de 2004 a noviembre de 2005

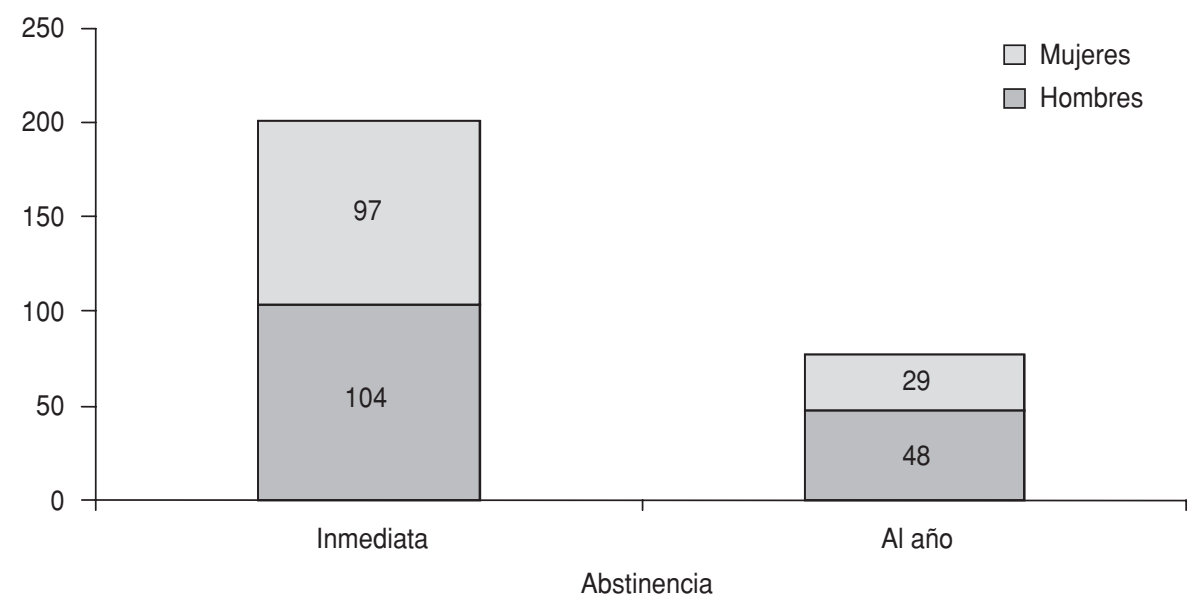

CUADRO 2. Frecuencia y asociación de algunos factores con la recidiva de tabaquismo al año de asistencia al Plan de Cinco Días para Dejar de Fumar, Rosario, Argentina, 2005

\begin{tabular}{|c|c|c|c|c|c|c|c|c|}
\hline \multirow[b]{2}{*}{ Factores } & \multicolumn{4}{|c|}{ Fuma al año (\%) } & \multirow[b]{2}{*}{ Total } & \multirow[b]{2}{*}{$\mathrm{RR}^{\mathrm{a}}$} & \multirow[b]{2}{*}{ IC $95 \%{ }^{b}$} & \multirow[b]{2}{*}{$P^{\mathrm{c}}$} \\
\hline & & $\mathrm{Si}$ & & No & & & & \\
\hline \multicolumn{9}{|l|}{ Género } \\
\hline Mujeres & 114 & $(79,7)$ & 29 & $(20,3)$ & 143 & 1,22 & $1,05-1,41$ & 0,005 \\
\hline Hombres & 90 & $(65,2)$ & 48 & $(34,8)$ & 138 & & & \\
\hline \multicolumn{9}{|l|}{ Edad } \\
\hline$\geq 40$ años & 147 & $(73,8)$ & 53 & $(26,2)$ & 200 & 1,04 & $0,88-1,22$ & 0,356 \\
\hline$<40$ años & 57 & $(70,4)$ & 24 & $(29,6)$ & 81 & & & \\
\hline \multicolumn{9}{|l|}{ Nivel educacional } \\
\hline Bajo & 29 & $(87,9)$ & 4 & $(12,1)$ & 33 & 1,07 & $0,87-1,33$ & 0,348 \\
\hline Alto & 175 & $(70,6)$ & 73 & $(29,4)$ & 248 & & & \\
\hline \multicolumn{9}{|l|}{ Edad de comienzo } \\
\hline$<20$ & 176 & $(70,4)$ & 74 & $(29,6)$ & 250 & 0,78 & $0,67-0,91$ & 0,018 \\
\hline$\geq 20$ & 28 & $(90,3)$ & 3 & $(9,7)$ & 31 & & & \\
\hline \multicolumn{9}{|l|}{ Consumo diario de tabaco } \\
\hline Más de 20 cigarrillos & 87 & $(73,7)$ & 31 & $(26,3)$ & 118 & 0,97 & $0,84-1,12$ & 0,416 \\
\hline 20 cigarrillos o menos & 117 & $(71,7)$ & 46 & $(28,3)$ & 163 & & & \\
\hline \multicolumn{9}{|l|}{ Años totales de consumo } \\
\hline$\geq 20$ años & 160 & $(73,3)$ & 58 & $(26,7)$ & 218 & 1,05 & $0,88-1,26$ & 0,330 \\
\hline$<20$ años & 44 & $(69,8)$ & 19 & $(30,2)$ & 63 & & & \\
\hline \multicolumn{9}{|c|}{ Nivel de dependencia a la nicotina } \\
\hline Alto & 62 & $(81,6)$ & 14 & $(18,4)$ & 76 & 1,17 & $1,01-1,35$ & 0,030 \\
\hline Bajo & 142 & $(69,3)$ & 63 & $(30,7)$ & 205 & & & \\
\hline \multicolumn{9}{|l|}{ Apoyo social } \\
\hline No tuvo & 49 & $(75,4)$ & 16 & $(24,6)$ & 65 & 1,05 & $0,89-1,23$ & 0,343 \\
\hline Sí tuvo & 155 & $(71,8)$ & 61 & $(28,2)$ & 216 & & & \\
\hline
\end{tabular}

a $R$ R: riesgo relativo.

b IC95\%: intervalo de confianza de $95 \%$.

c Para la prueba de la ji al cuadrado. Nivel de significación: 0,05.

que comenzaron a consumir tabaco en la adolescencia y que tenían un alto nivel educacional. En este estudio no se encontró asociación entre estas características y la eficacia del $\mathrm{P} 5 \mathrm{D}$, a pesar de que se ha indicado que el consumo temprano de tabaco (17) y el nivel de educación están asociados con una mayor tasa de abstinencia al año de abandonar el cigarrillo.
El moderado consumo promedio de cigarrillos en los participantes en el estudio (algo más de 20 cigarrillos diarios) no debe considerarse menos dañino. No existe un "umbral seguro" en el consumo de cigarrillos, aunque diversos estudios han demostrado que las enfermedades producidas por el consumo de cigarrillos aparecen más frecuentemente en las personas que consumen mayor cantidad de ellos (15, 18). No obstante, cuando se analiza este dato en relación con el éxito en la abstinencia o el abandono del tabaquismo, se observa que las personas que consumen mayor cantidad de cigarrillos al día y los que más tiempo llevan fumando son los que más dificultades experimentan para abandonar este hábito y, en consecuencia, los que tienen menos éxito en sus intentos de dejar el tabaquismo (16). En el presente estudio no se encontró una asociación significativa entre los años de fumador y la cantidad de cigarrillos consumidos con el éxito en la abstinencia al año.

La abstinencia observada en $27,4 \%$ de los participantes un año después de haber asistido al P5D se encuentra entre los valores de eficacia más elevados encontrados con este método (19, 20 ) y es superior a las eficacia de $16 \%$ informada en Francia (21) y a la de $25 \%$ observada en Suiza (22).

La comparación de la eficacia encontrada en este estudio con la de otros programas conductuales o cognitivos se hace difícil debido a la heterogeneidad de los métodos utilizados. Sin embargo, en un metaanálisis publicado recientemente se concluyó que la eficacia en los estudios que utilizaron la terapia de grupo varió entre $14,2 \%$ y $33,7 \%$ (23) y no se encontraron evidencias de que algún componente en particular de las terapias de grupo influyera más en la eficacia, excepto el apoyo y el entrenamiento de habilidades para la cesación. En general, las terapias de grupo resultaron más eficaces que las terapias individuales o de autoayuda (razón de posibilidades [OR]: 2,04; IC95\%: 1,60 a 2,60) (24).

La decisión voluntaria de participar en el P5D y la aproximación psicológica utilizada en este método ayuda- 
CUADRO 3. Causas más frecuentes de recaída en una muestra de los participantes en el Plan de Cinco Días para Dejar de Fumar en la ciudad de Rosario, Argentina, septiembre de 2004 a noviembre de 2005

\begin{tabular}{lcr}
\hline \multirow{2}{*}{ Causas } & \multicolumn{2}{c}{ Participantes } \\
\cline { 2 - 3 } & No. & $\%$ \\
\hline Síntomas del síndrome de abstinencia & 83 & 40,7 \\
Situaciones estresantes & 58 & 28,5 \\
Tabaquismo en el entorno personal & 15 & 7,3 \\
Falta de motivación & 18 & 8,8 \\
Otras & 30 & 14,7 \\
$\quad$ Total & 204 & 100,0 \\
\hline
\end{tabular}

ron al fumador a sentirse responsable de abandonar el tabaquismo. En este estudio no se confirmó la cesación del hábito mediante la medición de monóxido de carbono o de nicotina en la orina, ya que se consideró que la relación de confianza establecida entre los moderadores y cada uno de los participantes durante los cursos del P5D garantiza la veracidad de las respuestas a las preguntas de seguimiento. No obstante, la ausencia de una medición objetiva para verificar el resultado declarado por el fumador es una limitación de este estudio.

El nivel de dependencia a la nicotina evaluado mediante la prueba de Fagerström es un predictor del éxito en el abandono del hábito tabáquico. La puntuación promedio en la población de fumadores de este estudio fue de 5 puntos, ligeramente superior que la puntuación de 4 encontrada en otro estudio (25). Un bajo nivel de dependencia a la nicotina estuvo asociado con una mayor eficacia al año de terminar el P5D.

El P5D resultó ser más eficaz en los hombres que en las mujeres. Aunque existen antecedentes en este sentido (26), este resultado puede deberse a que el tipo de información y las herramientas conductuales utilizadas en el P5D son más fácilmente asimiladas por los hombres. La participación de otros profesionales del área de la salud, como psicólogos o psiquiatras, puede contribuir a actualizar las técnicas conductuales y las estrategias empleadas para ayudar mejor a las mujeres a controlar los síntomas del síndrome de abstinencia.

Los participantes en el P5D atribuyeron la mayoría de las recaídas a los síntomas del síndrome de abstinencia y al estrés. Estos síntomas, originados en el sistema nervioso central, pueden ser atenuados con cantidades controladas de nicotina o de otros fármacos, como el bupropión (27). El marco establecido por el P5D no permite realizar un interrogatorio individualizado sobre la historia médica, las tendencias depresivas o la presencia de otros síndromes en cada uno de los participantes, de manera de ajustar el tipo y la duración del tratamiento y el uso de medicamentos de reemplazo de la ni- cotina. Es posible que se puedan lograr mejores resultados si se adaptara el P5D para permitir breves interrogatorios a los participantes y animar a asistir a la consulta con su médico de familia a los participantes que se encuentren en riesgo de depresión u otros síntomas relacionados con el abandono del tabaquismo (28).

Aunque la ciudad de Rosario es la segunda ciudad más grande de Argentina, su población no es representativa de todo el país y no es posible extrapolar la información obtenida a toda la población argentina. Sin embargo, este estudio es el primero de su tipo en el país $\mathrm{y}$, además de reflejar el comportamiento de un sector de la población de la ciudad de Rosario, puede servir como base para otros estudios similares.

A pesar de las limitaciones expuestas, se puede concluir que el P5D es una herramienta eficaz de prevención secundaria del tabaquismo. En el presente estudio, su eficacia estuvo asociada con el sexo masculino y el nivel de dependencia a la nicotina.

Agradecimientos. Los autores expresan su especial agradecimiento a los doctores Daniel Yáñez, Marta Bina y Heidi Schulz y a la ingeniera Silvia Grenóvero por sus invaluables aportes en la corrección lingüística y el análisis estadístico. Este estudio contó con un subsidio de la Facultad de Ciencias de la Salud de la Universidad Adventista del Plata para el proyecto Eficacia del Plan de Cinco Días para Dejar de Fumar, de la Iglesia Adventista del Séptimo Día, en habitantes de la ciudad de Rosario, provincia de Santa Fe, Argentina.

\section{REFERENCIAS}

1. World Health Organization. The European report on tobacco control policy. WHO European ministerial conference for a tobacco-free Europe. Warsaw, Poland; 2002 February 18-19. Warsaw: World Health Organization; 2002.

2. Peto R. Smoking and death: the past 40 years and the next 40. Br Med J. 1994;309:937-9.

3. López AD. Measuring the health hazards of tobacco: commentary. Bull World Health Organ. 1999;77(1):82-3.
4. Organización Panamericana de la Salud. Tabaco o salud: situación en las Américas. Un informe de la Organización Panamericana de la Salud. Washington, D.C.: OPS; 1992. (Publicación Científica No. 536).

5. Sociedad Argentina de Cardiología. Prevalencia de los factores de riesgo coronario en una muestra de la población argentina. Estudio REDIFA (Relevamiento de los Distritos de la Sociedad Argentina de Cardiología de los
Factores de Riesgo Coronario). Rev Argent Cardiol. 2002;70:300-11.

6. Tartaguone JE. Rol del profesional de la salud en el control del hábito tabáquico. Rev Argent Cardiol. 2003;71(3):159-60.

7. World Health Organization. The tobacco epidemic: a global public health emergency. Geneva: WHO; 1996.

8. Vicario A. Prevalencia de los factores de riesgo en la población del Hospital Español: 
una aproximación al riesgo cardiovascular absoluto. Rev Argent de Cardiol. 2003;71:6.

9. McFarland JW, Gimbel HW, Donald WA, Folkemberg EJ. The five day program to help individuals stop smoking: a preliminary report. Conn Med. 1964;28:885-90.

10. Schlegel RP, Manske SR, Page A, d'Avernas JR. Update on the delayed effects of the "Five Day Plan to Stop Smoking". Prev Med. 1984; 13(3):320-2.

11. McFarland JW, Folkemberg EJ. How to stop smoking in five days. Englewood Cliffs, NJ: Prentice-Hall Inc; 1964.

12. Thompson D, Wilson T. Discontinuance of cigarette smoking: "natural" and with "therapy". A ten week and ten-month follow-up study of 298 adult participants in a Five Day Plan to Stop Smoking. J Am Med Assoc. 1966; 196(12):1048-52.

13. Serraino D, Franceschi S, Tassan M, Baron AE, Talamini R. Cigarette smoking recidivism after participation in the "Five-Day Plan to Stop Smoking" in Northeastern Italy. Prev Med. 1993;22(2):272-83.

14. Daniel WW. Organización y resumen de los datos. Datos agrupados: la distribución de frecuencias. En: Daniel WW. Bioestadística: base para el análisis de las ciencias de la salud. 3ra ed. México, D.F.: Editorial Limusa; 1993. Pp. 23-30.

15. Heatherton TF, Kozlowski LT, Frecker RC, Fagerstrom KO. The Fagerstrom test for nicotine dependence: a revision of the Fagerstrom
Tolerance Questionnaire. Br J Addict. 1991; 86(9):1119-27.

16. Barrueco Ferrero M, Torrecilla García M, Hernández Mezquita MA, Jiménez Ruiz CA, Morales Sánchez A, Alonso Díaz A, et al. Tobacco cessation: action-stage result as a predictor of successful long-term maintenance. Arch Bronconeumol. 2007;43(3):136-42.

17. Flores Mateo G, Morchón Ramosa S, Masuet Aumatella C, Carrillo Santistevea P, Manchón Walsha P, Ramón Torrilla JM. Edad de inicio en el consumo del tabaco como predictor de la deshabituación tabáquica. Aten Primaria. 2005;35:466-71.

18. Kamholz SL. Pulmonary and cardiovascular consequences of smoking. Clin Occup Environ Med. 2006;5(1):157-71.

19. Romand R. A cognitive strategy for stopping smoking. The Five Day Plan. En: Slama K, ed. Tobacco and health. New York: Plenum Press; 1999. Pp. 813-6.

20. Picardi A, Bertholdi S, Morosini P. Association between the engagement of relatives in a behavioural group intervention for smoking cessation and higher quit rate at 6-, 12- and 24month follow-ups. Eur Addiction Res. 2002;8: 109-17.

21. Romand R, Gourgou S, Sancho Garnier H. A randomized trial assessing the Five-Day Plan for Smoking Cessation. Addiction. 2005; 100(10):1546-54.

22. Frikart M, Etienne S, Cornuz J, Zellweger JP. Five-Day Plan for Smoking Cessation using group behaviour therapy. Swiss Med Wkly. 2003;133(3-4):39-43.

23. Stead LF, Lancaster T. Group behaviour therapy programmes for smoking cessation (Cochrane Review). In: The Cochrane Library. Issue 3. Oxford: Oxford Update Software; 2003.

24. Stead LF, Lancaster T. Group behaviour therapy programmes for smoking cessation. Cochrane Database Syst Rev. 2005;(2): CD001007.

25. West R. ABC of smoking cessation. Assessment of dependence and motivation to stop smoking. Br Med J. 2004;328:338-9.

26. Manchon Walsh P, Carrillo P, Flores G, Masuet C, Morchon S, Ramon JM. Effects of partner smoking status and gender on long term abstinence rates of patients receiving smoking cessation treatment. Addict Behav. 2007;32(1): 128-36.

27. Sampablo Lauro I, Angrill Paxeras J. Actualización en el tratamiento del tabaquismo. Arch Bronconeumol. 2004;40(3):123-32.

28. Jiménez Ruiz CA. Recomendaciones en el abordaje diagnóstico y terapéutico del tabaquismo. Documento de consenso. Arch Bronconeumol. 2003;39(1):35-41.

Manuscrito recibido el 27 de abril de 2006. Aceptado para publicación, tras revisión, el 7 de febrero de 2007.

ABSTRACT Objectives. To evaluate the effectiveness of the "Five-Day Plan to Stop Smoking" at the end of the intervention and after one year in a sample of participants in the city of Rosario, Santa Fe, Argentina.

\section{Effectiveness of the "Five-Day Plan to Stop Smoking" in a city in Argentina}

Methods. The quasi-experimental, longitudinal, prospective cohort, comparative study was based on the evaluation of the effectiveness of the "Five-Day Plan to Stop Smoking" in Rosario, Argentina, with eight groups of participants. Each session of the five-night course included presentations on medical aspects of smoking, an emphasis on group support in quitting, and relaxation techniques. The eight groups included a total of 739 people (50.5\% of them women and $49.5 \%$ men). Using a sample of 281 participants who were chosen at random, effectiveness was evaluated immediately after the end of each course and again after one year. Effectiveness was assessed in relation to the age the subjects began smoking, the average daily consumption of cigarettes, the number of years participants had smoked, their educational level, the number of previous attempts to quit smoking, and the level of nicotine dependence as measured by the Fagerström scale. Association among qualitative variables was determined using relative risk (RR), with $95 \%$ confidence intervals. Differences in the means of quantitative variables were determined using the $t$ test for independent samples. The chisquare test was used to establish differences among the categorical variables studied. Results. Of the 281 participants (138 men and 143 women) in the sample, $201(71.5 \%)$ (104 men and 97 women) quit smoking by the end of the course, and 77 (27.4\%) (48 men and 29 women) did not smoke after one year. There was a significant association between relapse and female gender $(R R=1.22 ; P=0.005)$, as was there for a score $\geq 7$ on the Fagerström scale of nicotine dependence ( $R R=1.17 ; P=0.03)$. Beginning to smoke before the age of 20 was a protective factor against relapse among those attending the "Five-Day Plan to Stop Smoking" courses. The most frequent determinants of relapse were abstinence syndrome symptoms (40.3\%) and stressful situations (28.2\%).

Conclusions. The "Five-Day Plan to Stop Smoking" is an effective tool for secondary prevention of smoking. In the study population its effectiveness was associated with male gender and with a lower level of nicotine dependence.

Key words Smoking cessation, Argentina. 